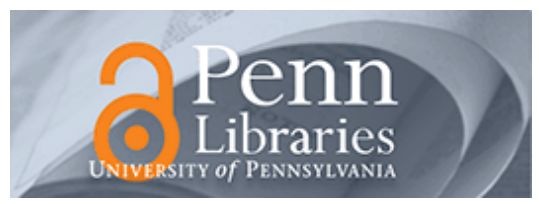

University of Pennsylvania ScholarlyCommons

December 2007

\title{
Piezoresponse imaging of lead zirconate titanate microfibers and numerical analysis of its electric field distribution
}

Yu Wang

University of Pennsylvania

Jorge J. Santiago-Aviles

University of Pennsylvania, santiago@seas.upenn.edu

Follow this and additional works at: https://repository.upenn.edu/ese_papers

\section{Recommended Citation}

Yu Wang and Jorge J. Santiago-Aviles, "Piezoresponse imaging of lead zirconate titanate microfibers and numerical analysis of its electric field distribution", . December 2007.

Copyright 2007 American Institute of Physics. This article may be downloaded for personal use only. Any other use requires prior permission of the author and the American Institute of Physics. Reprinted in Applied Physics Letters, Volume 91, Article 263108, December 2007, 3 pages.

Publisher URL: http://dx.doi.org/10.1063/1.2827564

This paper is posted at ScholarlyCommons. https://repository.upenn.edu/ese_papers/319

For more information, please contact repository@pobox.upenn.edu. 


\title{
Piezoresponse imaging of lead zirconate titanate microfibers and numerical analysis of its electric field distribution
}

\author{
Abstract \\ Piezoresponse imaging technique was transplanted from thin film to probe polarization domains and \\ local properties in single electrospun lead zirconate titanate microfibers. The corresponding electric field \\ distribution was numerically analyzed. The biased conic tip is found to produce a field that peaks on its \\ apex and decreases rapidly toward the bottom metal electrode. A strong field exists only in a thin surface \\ region and cannot pole the affected domain even with its magnitude of $10^{8} \mathrm{~V} / \mathrm{m}$ on the fiber surface.

\section{Comments} \\ Copyright 2007 American Institute of Physics. This article may be downloaded for personal use only. Any \\ other use requires prior permission of the author and the American Institute of Physics. Reprinted in \\ Applied Physics Letters, Volume 91, Article 263108, December 2007, 3 pages. \\ Publisher URL: http://dx.doi.org/10.1063/1.2827564
}




\title{
Piezoresponse imaging of lead zirconate titanate microfibers and numerical analysis of its electric field distribution
}

\author{
Yu Wang ${ }^{\text {a) }}$ and Jorge Santiago-Avilés \\ Department of Electrical and Systems Engineering, University of Pennsylvania, 200 South 33rd Street, \\ Philadelphia 19104, USA
}

(Received 10 September 2007; accepted 1 December 2007; published online 28 December 2007)

\begin{abstract}
Piezoresponse imaging technique was transplanted from thin film to probe polarization domains and local properties in single electrospun lead zirconate titanate microfibers. The corresponding electric field distribution was numerically analyzed. The biased conic tip is found to produce a field that peaks on its apex and decreases rapidly toward the bottom metal electrode. A strong field exists only in a thin surface region and cannot pole the affected domain even with its magnitude of $10^{8} \mathrm{~V} / \mathrm{m}$ on the fiber surface. () 2007 American Institute of Physics. [DOI: 10.1063/1.2827564]
\end{abstract}

Lead zirconate titanate, $\mathrm{Pb}\left(\mathrm{Zr}_{x} \mathrm{Ti}_{1-x}\right) \mathrm{O}_{3}(\mathrm{PZT})$, is a wellknown ferroelectric material with significant technological importance. ${ }^{1}$ PZT fibers have potential for utilization in high performance hydrophones and ultrasonic transducer applications. ${ }^{2}$ We synthesized PZT fibers with diameter from $100 \mathrm{~nm}$ to $20 \mu \mathrm{m}$ by means of electrospinning and metalloorganic decomposition. ${ }^{3,4}$ Since then, it has been a challenge for us to evaluate their ferroelectric properties. Usually, PZT is evaluated as dielectric media in a sandwiched metal/PZT/ metal capacitor. ${ }^{5}$ Such evaluation does not work for a single PZT fiber with diameter less than $10 \mu \mathrm{m}$ because of its ultralow capacitance. Recently, piezoresponse imaging (PRI) has been developed to probe polarization domains in ferroelectric thin films (including PZT thin film), measure their properties in micro- or nano-scale, and correlate domain polarization and local properties directly with topography and morphology. ${ }^{6,7}$

PRI is realized through the combination of scanning probe microscopy (SPM) and lock-in electrical measurement techniques. ${ }^{6,7}$ It makes use of a sandwiched conductive SPM tip/PZT thin film/bottom metal capacitor, where the vertical SPM tip acts as the top electrode that can be precisely positioned and moved in a program-controlled way. dc and ac voltages $\left[V_{\mathrm{dc}}\right.$ and $\left.V_{\mathrm{ac}} \cos (\omega t)\right]$ are applied simultaneously between the tip and the bottom metal,

$$
V_{\text {tip }}=V_{\mathrm{dc}}+V_{\mathrm{ac}} \cos (\omega t)
$$

set up electric field $(E)$ and induce piezoelectric oscillation $(\delta)$ on PZT surface,

$\delta=\delta_{0}+\delta_{1} \cos \left(\omega t+\phi_{1}\right)+\delta_{2} \cos \left(2 \omega t+\phi_{2}\right)+\cdots \cdots$,

which is actually detected by SPM as a voltage signal $(V)$,

$V=V_{0}+V_{1} \cos \left(\omega t+\varphi_{1}\right)+V_{2} \cos \left(2 \omega t+\varphi_{2}\right)+\cdots \cdots$,

where the subscript $i(=0,1,2, \ldots)$ stands for the $i$ th harmonic component, $\phi_{i}-\varphi_{i}$ is the $i$ th harmonic phase shift of the SPM detecting system, phase $\phi_{1}=0$ and $\pi$ when the polarization vector $(\mathbf{P})$ is up and down in the local domain. Of the harmonic components of various orders, the dc or static components reflect the surface topography if $V_{\mathrm{dc}}=0$; the $1 \mathrm{st}$ harmonic component reflects local piezoelectric properties,

\footnotetext{
${ }^{a)}$ Electronic mail: wangyu@ @eas.upenn.edu.
}

$$
\delta_{1}=d_{33} V_{\mathrm{ac}},
$$

where $d_{33}$ is the local piezoelectric constant. $V_{1}, \delta_{1}$, and $d_{33}$ are proportional to the magnitude of polarization $(P)$ and their sign reflects the $\mathbf{P}$ direction. With $V_{\mathrm{dc}}=0, \mathbf{P}$ is the spontaneous polarization $\mathbf{P}_{\mathbf{s}}$. For each tip position, if $V_{\mathrm{dc}}$ is swept and $d_{33}$ is measured, $d_{33}$ can be plotted against $V_{\mathrm{dc}}$ as a local piezoelectric curve. $^{7}$

The local piezoelectric curve is not the same as the better-known polarization ( $P$ versus $E$ ) curve in macroscale. Usually, $E$ is considered to be more pertinent than $V_{\mathrm{dc}}$ for properties characterization. However, $d_{33}$ has not been plotted against $E$, mainly due to the difficulties in calculating $E$ from $V_{\mathrm{dc}}$. The actual interaction between the SPM tip and PZT thin film has been modeled using the layer capacitor model, the method of image charges, and the finite element analysis (FEA). ${ }^{7}$ In these cases, the approximate azimuthal symmetry reduces the problem into two dimensions. ${ }^{8}$

PRI has not been applied to fibers. In this paper, we try to use it to probe electrospun PZT microfibers and to numerically analyze its field distribution, which will help to understand our PRI and local measurement results. An example is to convert $V_{\mathrm{dc}}$ into $E$ and present the local piezoelectric curve as $d_{33}$ against $E$. We use a structure of vertical conductive SPM tip/horizontal PZT fiber/bottom metal contact [Fig. 1(a)]. Since the replacement of a thin film by a fiber breaks the azimuthally symmetry, the problem is solved in three dimensions (3D).

The potential $(u)$ between the biased tip and the grounded bottom metal is governed by the Laplace equation

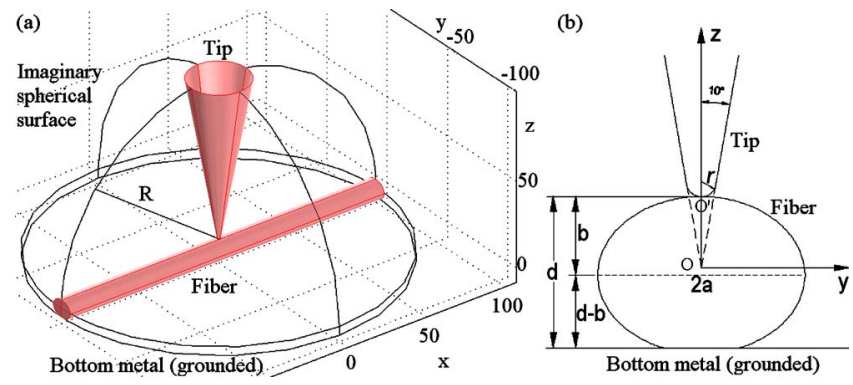

FIG. 1. (Color online) (a) 3D geometric model of the problem and (b) cross section of the tip and the fiber. 

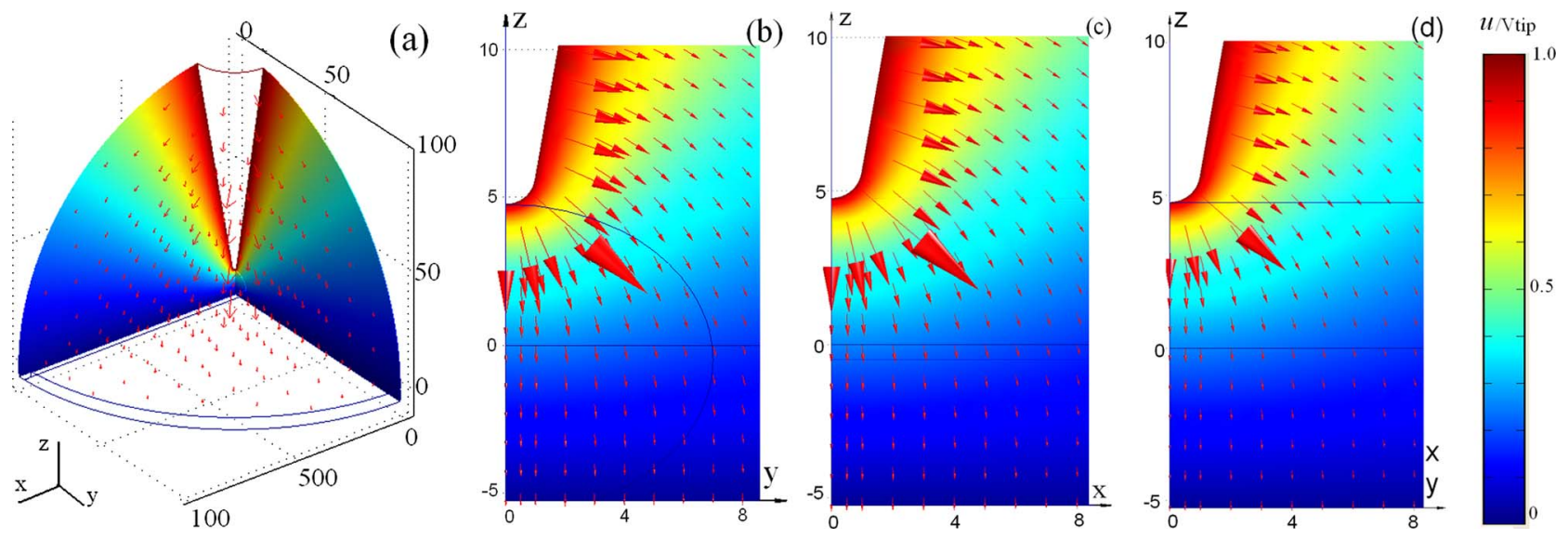

FIG. 2. (Color online) Potential and field distributions (a) in 3D, on the cross section (b) parallel and (c) perpendicular to the PZT fiber axis, and (d) on the cross section of a PZT thin film.

$$
\nabla^{2} u=0,
$$

with Dirichelet boundary conditions, ${ }^{9}$

$$
\begin{aligned}
& u=V_{\text {tip }}, \\
& u=0, \\
& u=0,
\end{aligned}
$$

on the tip surface, on the grounded metal, and at infinity, respectively. The tip is approximated as an infinite rounded cone with full cone angle of $20^{\circ}$ and apex radius of curvature $r$ (=35 nm for our tip). Our previously SPM topography measurement ${ }^{4}$ showed that the fiber has an elliptic cross section (with its semimajor and minor axes $a$ and $b$ ) except that its bottom is flat (as if cut slightly) and it contacts the bottom metal over a small region. Accordingly, the vertical dimension of the fiber $d$ is slightly less than $2 b$ [Fig. 1(b)]. Additionally, we set the dielectric constant of PZT fiber to be 100 .

Since FEA requires a finite boundary and we are concerned mostly about the potential and field distribution between the tip and the bottom metal contact, we replace the infinite boundary by an imaginary spherical surface centered at the virtual end of the tip cone if it is not rounded $(\mathrm{O})$ with a large radius $R=100 r \gg a, b$, and $d$ (Fig. 1). On such a large spherical surface, the radial equipotentials or Neumann boundary condition,

$$
\frac{\partial u}{\partial n}=0,
$$

is a good approximations. Equation (5), with Eqs. (6a), (6b),
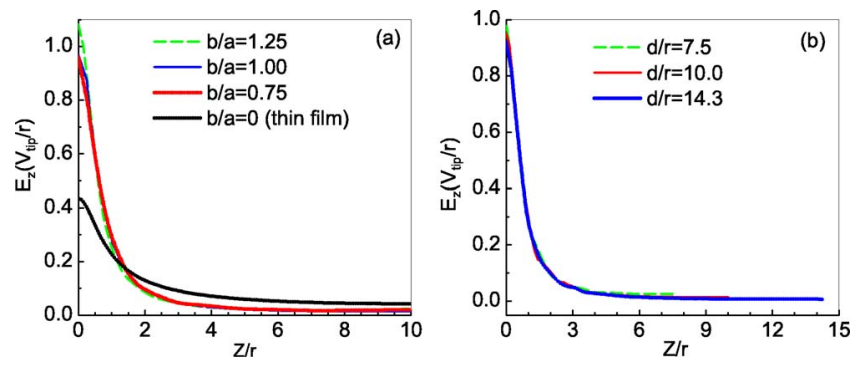

FIG. 3. (Color online) Effect of (a) cross-sectional shape ( $b / a$ value) and (b) thickness of the fiber on $E$ distribution along $z$ axis between the tip and the

bottom metal. $Z$ is the distance from $\mathrm{O}^{\prime}$ along the $-z$ direction. phase images of a single PZT microfiber (the area size is $10 \times 10 \mu \mathrm{m}^{2}$ ).
Downloaded 13 Feb 2008 to 130.91 .116 .168 . Redistribution subject to AlP license or copyright; see http://apl.aip.org/apl/copyright.jsp and $\left(6 c^{\prime}\right)$, were solved using FEA embedded in FEMLAB. Thanks to the mirror symmetry with respect to the $y o z$ and zox planes, the problem was solved in a quarter of the geometry.

Figure 2 presents the $u$ and $E$ distributions for a typical case with $d=10 r$ and $a=7 r, b=5.25 r$, as well as for a thin film with the same vertical size $d$. Figure 3 shows the effect of the elliptic cross-sectional shape (the $b / a$ ratio) and the cross-sectional size $(a, b$, and $d$ in proportion, given $b / a=0.70)$ on the $E$ distribution along the $z$ axis between the tip and the metal with $d=10 r, b=5.25 r$, and $a=4 r, 5 r, 7 r$, and 0 (thin film). For all fiber cases, the $E$ distribution is similar: it peaks on the tip apex $\left(\mathrm{O}^{\prime}\right)$ and decreases drastically toward the bottom metal. Despite the size and shape variation on the fiber's cross section, the field distribution remains reasonably unaffected around $\mathrm{O}^{\prime}$ with its peak,

$$
E_{\max } \approx 1.0 V_{\text {tip }} / r .
$$

Equation (7) clearly indicates that the effects of fiber size and shape are overwhelmed by the field-concentrating effect of the tip's conic shape ${ }^{9}$ even though the tip is rounded. Toward the bottom metal, the field-concentrating effect diminishes and the flat bottom metal starts to take effect. The $E_{\max }$ value is more than twice as at the thin film surface. However, the difference decreases rapidly and changes its sign around $d / 10$ below the surface.

PRI was conducted on electrospun PZT microfibers using a Dimension ${ }^{\mathrm{TM}} 3100$ SPM by Digital Instruments under the conditions: $V_{\mathrm{dc}}=0 \mathrm{~V}, V_{\mathrm{ac}}=10 \mathrm{~V}, \omega=140 \pi \mathrm{kHz}$. Figures 4 and 5 show typical SPM topography and PRI micrographs of a single fiber about $5 \mu \mathrm{m}$ in diameter and an area within that fiber. Despite of the continuous surface topography [Figs. 4(a) and 5(a)], PRI micrographs reveal numerous spontaneous polarization domains of size from

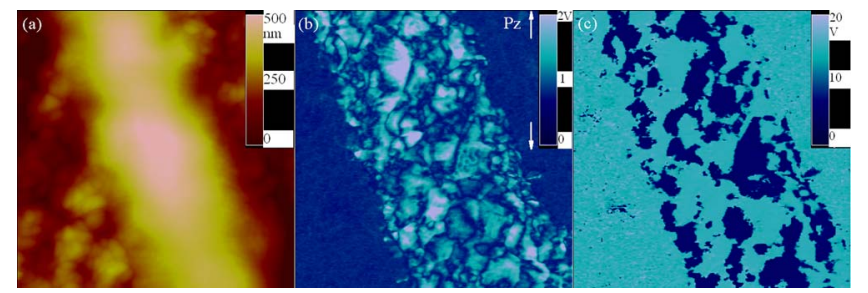

FIG. 4. (Color online) (a) SPM topography, (b) PRI amplitude, and (c) PRI phase images of a single PZT microfiber (the area size is $10 \times 10 \mu \mathrm{m}^{2}$ ). 


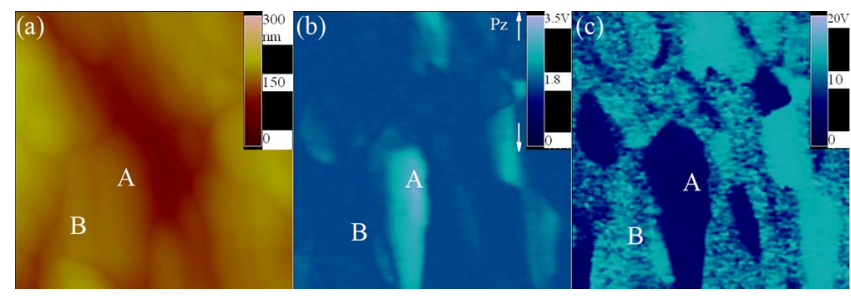

FIG. 5. (Color online) (a) SPM topography, (b) PRI amplitude, and (c) PRI phase images of a $1.5 \times 1.5 \mu \mathrm{m}^{2}$ area within the PZT microfiber.

100 to $1000 \mathrm{~nm}$ [Figs. 4(b), 4(c), 5(b), and 5(c)]. Their bright, dark, and grey colors correspond to up, down, and horizontal polarization directions, respectively.

During our local measurement, the SPM tip was positioned on two neighboring domains with opposite polarization direction (Fig. 5) and $V_{\mathrm{dc}}$ was swept in a cyclic way of $0 \mathrm{~V} \rightarrow 10 \mathrm{~V} \rightarrow-10 \mathrm{~V} \rightarrow 0 \mathrm{~V}$ with a step of $\pm 0.1 \mathrm{~V}$. Figure 6 presents local piezoelectric curves as $d_{33}$ against $V_{\mathrm{dc}}$ and $E_{\max }$ using Eq. (7) with $r=35 \mathrm{~nm}$. Note that $d_{33}$ keeps its sign in both curves, implying that the polarization was not switched in two domains. Although $E_{\max }$ is as high as $2.9 \times 10^{8} \mathrm{~V} / \mathrm{m}, E$ diminishes rapidly toward the bottom metal.

In summary, PRI technique was used to probe polarization domains and local properties in a PZT microfiber. Its electric field distribution was numerically solved. The field is found to decrease rapidly from its maximum at the tip apex toward the bottom metal. The strong field, mainly produced by the tip's conic shape, exists only in a surface region and cannot pole the affected domain even with an order of magnitude of $10^{8} \mathrm{~V} / \mathrm{m}$ at fiber surface.
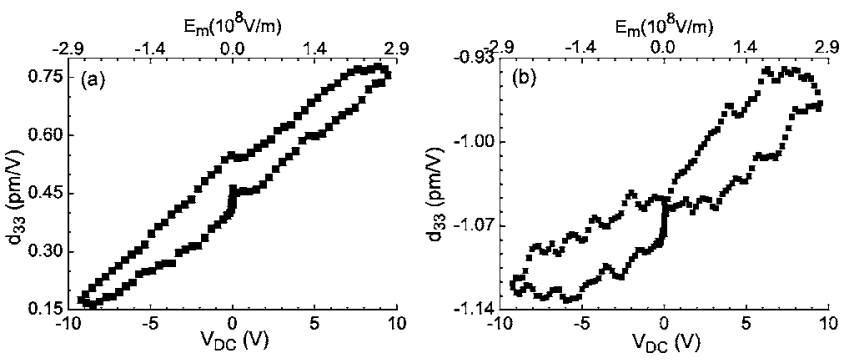

FIG. 6. Local $d_{33}$ vs. $V_{\mathrm{dc}}$ curves of domains (a) A and (b) B in Fig. 5.

We would like to thank Professor D. Bonnell's group with the Laboratory for Research on the Structure of Matter at the University of Pennsylvania for their help with the PRI experiments. This work was sponsored in part by NSFDMR-0353730 and by Penn's NBIC program (NSF-DMR0425780).

${ }^{1}$ T. M. Shaw, S. Trolier-McKinstry, and P. C. McIntryre, Annu. Rev. Mater. Sci. 30, 263 (2000).

${ }^{2}$ S. Yoshikawa, U. Selvaraj, P. Moses, J. Withams, R. Meyer, and T. Shrout, J. Intell. Mater. Syst. Struct. 6, 152 (1995).

${ }^{3}$ Y. Wang, S. Serrano and J. J. Santiago-Avilés, Mater. Res. Soc. Symp. Proc. 702, 359 (2002).

${ }^{4}$ Y. Wang and J. J. Santiago-Avilés, Nanotechnology 15, 32 (2004).

${ }^{5}$ S. Trolier-Mckinstry and P. Muralt, J. Electroceram. 12, 7 (2004).

${ }^{6}$ H. Birk, J. Glatz-Reichenbach, L. Jie, E. Schreck, and K. Dransfeld, J. Vac. Sci. Technol. B 9, 1162 (1991).

${ }^{7}$ C. Harnagea, "Local piezoelectric response and domain structures in ferroelectric thin films investigated by voltage-modulated force microscopy," Ph.D. thesis, 2001; http://sundoc.bibliothek.uni-halle.de/diss-online/01/ 01H318, June 23, 2007.

${ }^{8}$ S. Lanyi, J. Torok, and P. Rehurek, J. Vac. Sci. Technol. B 14, 892 (1996). ${ }^{9}$ J. D. Jackson, Classical Electrodynamics, 3rd ed. (Wiley, New York, 1999). 